\title{
Deformation Dynamics and Young's Modulus of Silver Nanocontacts*
}

\author{
Hideki Masuda and Tokushi Kizuka ${ }^{\dagger}$ \\ Institute of Materials Science, University of Tsukuba, Tsukuba, Ibaraki 305-8573, Japan
}

(Received 10 November 2008; Accepted 22 February 2009; Published 16 May 2009)

\begin{abstract}
We observed the tensile deformation process of silver nanocontacts (Ag NCs) in situ at room temperature by high-resolution transmission electron microscopy. Simultaneously, the conductance and the tensile force of the NCs were measured. Stress-strain relationship of Ag NC was investigated. Typical saw-tooth curves showing elastic and plastic deformations of NCs were observed. The Young's modulus of NCs was estimated from the stress-strain curve. [DOI: 10.1380/ejssnt.2009.621]
\end{abstract}

Keywords: Transmission Electron Microscopy; Silver; Nanocontact

\section{INTRODUCTION}

Metallic nanocontacts (NCs) exhibit intriguing electric properties for next-generation nanometer-sized devices, i.e., ballistic conduction and the quantized conductance defined by a quantum unit $\left(G_{0}=2 e^{2} / h\right.$, where $e$ is the electron charge and $h$ is Planck's constant) [1-18]. Metallic NCs are produced by contact of two nanometer-sized tips and are thinned by subsequent breaking. Thus, the structure of metallic NCs depends on their deformation characteristics. Whereas much effort by simulation has been directed for understanding the atomistic deformation dynamics of metallic NCs, in situ high-resolution transmission electron microscopy (HRTEM) has taken a prominent role in the direct observation of the dynamics $[17,19-24]$. The atomistic dynamics of NCs of only limited metallic elements, such as gold, palladium, and iridium, has been observed [25-27]. In this report, we focus on silver $(\mathrm{Ag}) \mathrm{NCs}$ and investigate their tensile deformation by in situ HRTEM with subnanonewton force measurement.

\section{EXPERIMENTAL}

The experimental method in this study was developed on the basis of in situ HRTEM combined with subnanonewton force measurements used in atomic force microscopy (AFM) and electronic conductance measurements used in scanning tunneling microscopy [19, 23]. The system is illustrated in Fig. 1. First, we prepared nanometer-sized Ag tips: Ag was evaporated in a vacuum chamber and deposited on a Si cantilever with a nanotip for AFM. The spring constant of the cantilever was $5.3 \pm 0.3 \mathrm{~N} / \mathrm{m}$. The cantilever tip was attached to the front of a tube piezoelement on a cantilever holder for HRTEM. A Ag plate of $0.2 \mathrm{~mm}$ thickness was attached to the second plate holder. The contact edge of the plate for contact was thinned to $5-20 \mathrm{~nm}$ by argon ion milling. The cantilever and plate holders were then inserted into the $i n$ situ transmission electron microscope at the University of

\footnotetext{
* This paper was presented at International Symposium on Surface Science and Nanotechnology (ISSS-5), Waseda University, Japan, 9-13 November, 2008.

†Corresponding author: kizuka@ims.tsukuba.ac.jp
}

Tsukuba. The specimen chamber of the microscope was evacuated first by a turbomolecular pump and then by an ion pump, resulting in a vacuum of $1 \times 10^{-5} \mathrm{~Pa}$. The cantilever tip was brought into contact with the edge surface of the opposing plate by piezomanipulation while applying a bias voltage of $65 \mathrm{mV}$. The cantilever tip was then pressed into the plate to prepare NCs and then retracted to elongate them. A series of these manipulations was performed at room temperature. The structural dynamics during the process was observed in situ by lattice imaging by HRTEM using a television capture system. The time resolution of image observations was $17 \mathrm{~ms}$. The force acting on NCs was simultaneously measured by the optical detection of cantilever deflection. The electrical conductance was measured using a two-terminal method. The high-resolution images and detected signals in this sys-

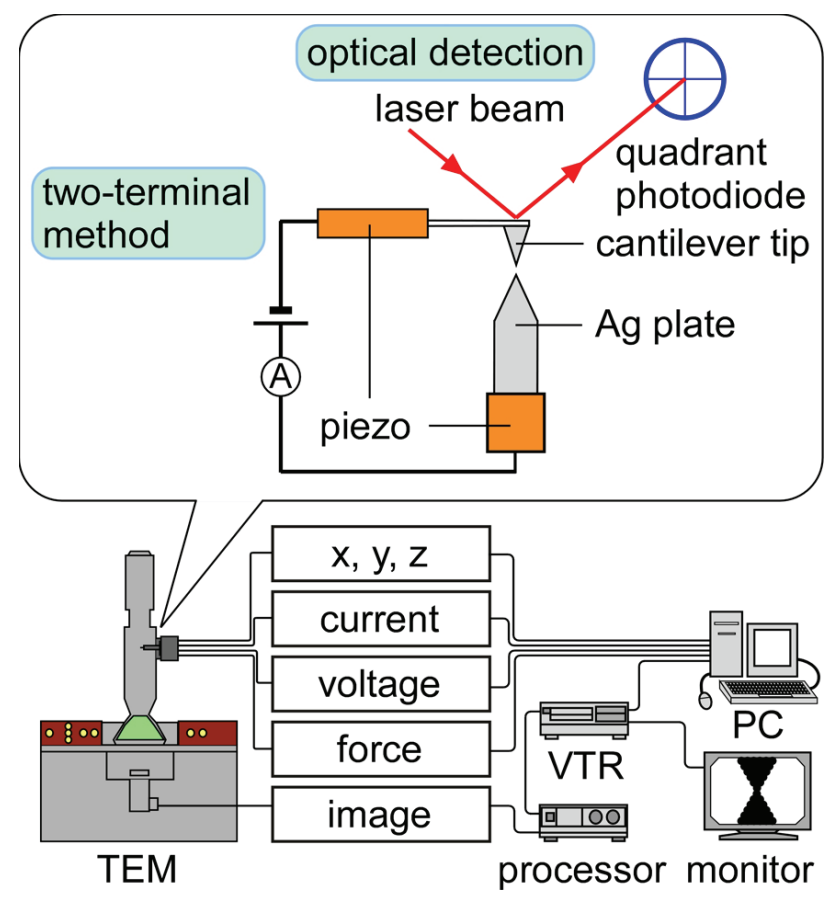

FIG. 1: Illustration of in situ transmission electron microscope (TEM) in this study. The voltages applied on piezoelement for specimen displacement along the $x, y, z$ directions, the current through and bias voltage applied to nanocontacts, the force acting on nanocontacts, and images are recorded using a computer. 

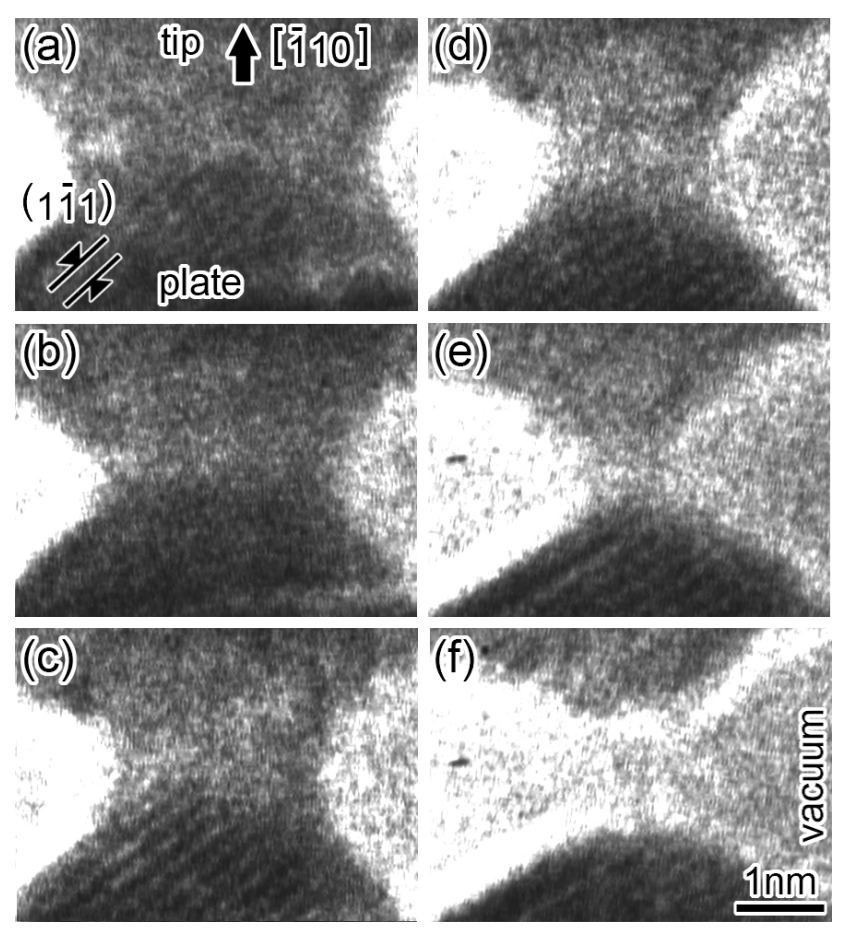

FIG. 2: Time-sequence series of high-resolution images of tensile deformation process of $\mathrm{Ag}$ nanocontact.

tem were simultaneously recorded and analyzed for every image.

\section{RESULTS AND DISCUSSION}

Figure 2 shows a time-sequence series of high-resolution images of a tensile deformation process of a $\mathrm{Ag} \mathrm{NC}$. The retraction speed was $1.6 \mathrm{~nm} / \mathrm{s}$. The tip and the plate are observed as dark areas in the upper and the lower sides in Figs. 2(a)-2(f), respectively. The lattice fringes of the (111) planes were imaged on the plate, as shown in Fig. 2. This plate was negatively biased by $65 \mathrm{mV}$ to the tip. In the middle of Fig. 2(a), the contact of approximately $3.5 \mathrm{~nm}$ width is located. Since both the edge surfaces of the tip were parallel to those of the plate, as shown in Fig. 2(d), the orientation of the tip and plate are identified to be the same. As the tip was retracted from the plate along [110], as indicated by the arrow in Fig. 2(a), the NC was thinned gradually, as shown in Figs. 2(a)-2(e). In Fig. 2(e), the contact width decreased to several-atoms width. The contact finally broke, as shown in Fig. 2(f). The slip occurred on the (111) planes along [011]. This slip system is typical one in face-centered-cubic metals. We focused on the mechanical properties of the NC shown in Fig. 2.

Figure 3 shows variations in the strain $(\varepsilon)$, the minimum cross-sectional area of the $\mathrm{NC}(S)$, the force $(F)$ and stress $(\sigma)$ applied on the $\mathrm{NC}$, the current $(I)$ and current density $(\rho)$ through the NC during tensile deformation presented in Fig. 2 as function of time. To estimate $\varepsilon$, we defined two stationary points beside the $\mathrm{NC}$ [23]. The distance between the two points at time a in Fig. 3 was selected to $4.5 \mathrm{~nm}$. The fluctuation in the tip-

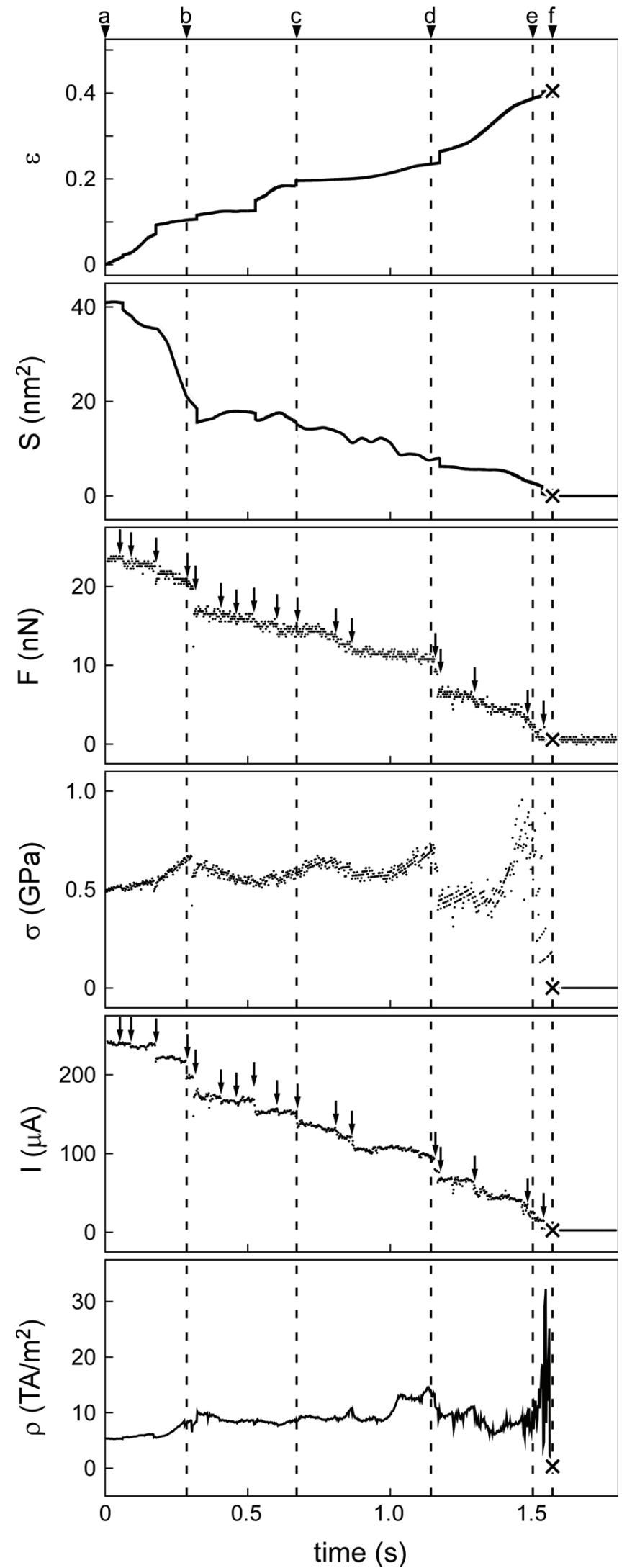

FIG. 3: Variations in strain $(\varepsilon)$, minimum cross-sectional area $(S)$, force $(F)$, stress $(\sigma)$, current $(I)$, and current density $(\rho)$ of $\mathrm{Ag}$ nanocontact during tensile deformation observed in Fig. 2 as function of time. Typical slip events are indicated by the arrows in force and current variations. 


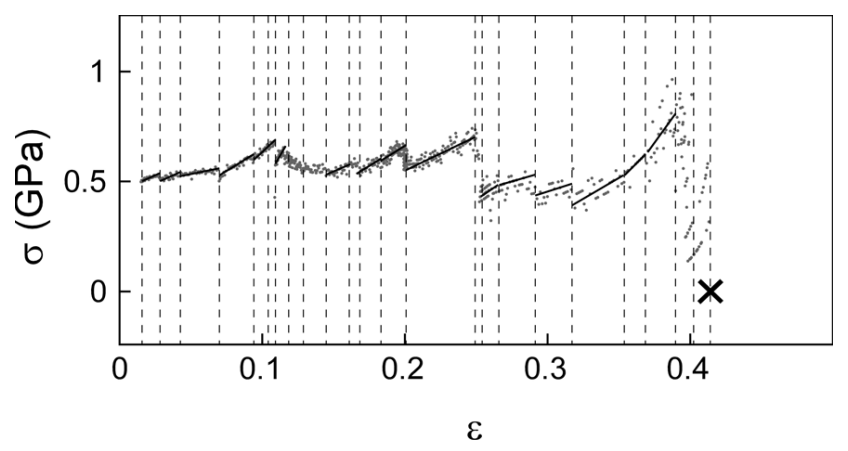

FIG. 4: The stress-strain relationship of Ag nanocontact observed in Fig. 2.

plate distance at a piezovoltage of zero was comparable to the spatial resolution of the observation, as a result of which the error of the estimated strain and stress was 0.01 and $0.05 \mathrm{GPa}$, respectively. Times a-f in Fig. 3 correspond to those of the images in Figs. 2(a)-2(f). We assumed that the cross-sectional area of the $\mathrm{NC}$ at its minimum width was circular, and calculated the area from the width measured in the images. The strain increased gradually as time passed, and in contrast, the minimum cross-sectional area decreased with time. The NC was thinned to the several atoms at time e, as observed in Fig. 2(e). We calculated the stress and the current density by dividing the force and the current by the minimum cross-sectional area, respectively. Slip events occurred at discrete decreases in both the force and stress. Simultaneously, the current showed rapid variations, as seen in Fig. 3.

Figure 4 shows a stress-strain relationship of the $\mathrm{Ag}$ $\mathrm{NC}$ observed in Fig. 2. As the strain increased, the stress showed a saw-tooth variation corresponding to a typical cycle of elastic elongation and slip. In Fig. 4, the cycles are demarcated by the dashed lines. The NC structure changed for each cycle. The stress at each edge of the saw-tooth implies the yield stress for each structure; the value is $\sim 0.5 \mathrm{GPa}$. The increase in strain in each cycle corresponds to the elastic limit of each structure. The elastic limit ranged from 0.005 to 0.05 . We estimated the Young's modulus for each cycle to be from $1.3 \pm 0.6$ to $9.0 \pm 6.2 \mathrm{GPa}$, which decreases by at least $90 \%$ from that of $\mathrm{Ag}$ bulk $~ 80 \mathrm{GPa}$. Before breaking, around a strain of $\sim 0.38$, the Young's modulus increased. It is suggested that the bonding nature of the NCs changes owing to size reduction to several atoms.

\section{CONCLUSIONS}

We investigated the variation in the structure of $\mathrm{Ag}$ $\mathrm{NCs}$, and the force acting on and conductance through them during tensile deformation. The yield stress, elastic limit and Young's modulus were estimated to be $0.5 \mathrm{GPa}$, 0.005-0.05, and 1.3-9.0 GPa, respectively, revealing that the mechanical properties of Ag NCs are considerably different from that of coarse-grained Ag crystals. The mechanical constants measured in this study implies that $\mathrm{Ag}$ NCs are stable when they are assembled into LSI.

\section{Acknowledgments}

This work was partly supported by funds for the special Research Project on Nanoscience, the University Research Projects of the University of Tsukuba, the Advanced Processing Mechanical Techniques Foundation, The Ogasawara Foundation for the Promotion of Science and Engineering, Shimadzu Science Foundation, and Grants-in-Aids from the Ministry of Education, Culture, Sports, Science and Technology, Japan (Nos. 18310075 and 19651047).
[1] C. J. Muller, J. M. van Ruitenbeek, and L. J. de Jongh, Phys. Rev. Lett. 69, 140 (1992).

[2] N. Agraï, J. G. Rodrigo, and S. Vieira, Phys. Rev. B, 47, 12345 (1993).

[3] J. M. Krans, C. J. Muller, I. K. Yanson, T. C. M. Govaert, R. Hesper, and J. M. van Ruitenbeek, Phys. Rev. B 48, 14721 (1993).

[4] J. I. Pascual, J. Méndez, J. Gómez-Herrero, A. M. Baró, N. García, and V. T. Binh, Phys. Rev. Lett. 71, 1852 (1993).

[5] G. Rubio, N. Agraït, and S. Vieira, Phys. Rev. Lett. 76, 2302 (1996).

[6] J. L. Costa-Krämer, N. García, P. García-Mochales, P. A. Serena, M. I. Marqués, and A. Correia, Phys. Rev. B 55, 5416 (1997).

[7] E. Scheer, N. Agraït, J. C. Cuevas, A. L. Yeyati, B. Ludoph, A. Martín-Rodero, G. R. Bollinger, J. M. Ruitenbeek, and C. Urbina, Nature 394, 154 (1998).

[8] A. I. Yanson, G. R. Bollinger, H. E. van den Brom, N. Agraït, and J. M. van Ruitenbeek, Nature 395, 783 (1998).

[9] S. K. Nielsen, M. Brandbyge, K. Hansen, K. Stokbro, J.
M. van Ruitenbeek, and F. Besenbacher, Phys. Rev. Lett. 89, 066804 (2002).

[10] C. Untiedt, A. I. Yanson, R. Grande, G. Rubio-Bollinger, N. Agraït, S. Vieira, and J. M. van Ruitenbeek, Phys. Rev. B 66, 085418 (2002).

[11] U. Landman, W. D. Luedtke, N. A. Burnham, and R. Colton, Science 248, 454 (1990).

[12] M. Brandbyge, J. Schiøtz, M. R. Sørensen, P. Stoltze, K. W. Jacobsen, J. K. Nørskov, L. Olesen, E. Laegsgaard, I. Stensgaard, and F. Besenbacher, Phys. Rev. B 52, 8499 (1995).

[13] A. M. Bratkovsky, A. P. Sutton, and T. N. Todorov, Phys. Rev. B 52, 5036 (1995).

[14] U. Landman, W. D. Luedtke, B. E. Salisbury, and R. L. Whetten, Phys. Rev. Lett. 77, 1362 (1996).

[15] A. G. Scherbakov, E. N. Bogachek, and U. Landman, Phys. Rev. B 53, 4054 (1996).

[16] A. Stalder and U. Dürig, J. Vac. Sci. Technol. B 14, 1259 (1996).

[17] A. Correia and N. García, Phys. Rev. B 55, 6689 (1997).

[18] H. Yasuda and A. Sakai, Phys. Rev. B 56, 1069 (1997).

[19] T. Kizuka, K. Yamada, S. Deguchi, M. Naruse, and N. 
Tanaka, Phys. Rev. B 55, R7398 (1997).

[20] T. Kizuka, Phys. Rev. Lett. 81, 4448 (1998).

[21] T. Kizuka, Phys. Rev. B 57, 11158 (1998).

[22] H. Ohnishi, Y. Kondo, and K. Takayanagi, Nature 395, 780 (1998)

[23] T. Kizuka, H. Ohmi, T. Sumi, K. Kumazawa, S. Deguchi, M. Naruse, S. Fujisawa, S. Sasaki, A. Yabe, and Y. Enomoto, Jpn. J. Appl. Phys. 40, L170 (2001).
[24] T. Kizuka, S. Umehara, and S. Fujisawa, Jpn. J. Appl. Phys. 40, L71 (2001).

[25] T. Kizuka, Phys. Rev. B 77, 155401 (2008).

[26] T. Matsuda and T. Kizuka, Jpn. J. Appl. Phys. 46, 4370 (2007).

[27] M. Ryu and T. Kizuka, Jpn. J. Appl. Phys. 45, 8952 (2006). 